\title{
Naturally Occurring Variants of Satellite Tobacco Mosaic Virus
}

\author{
D. M. Mathews and J. A. Dodds
}

Department of Plant Pathology, University of California, Riverside 92521.

Accepted for publication 16 February 1998.

\section{ABSTRACT}

Mathews, D. M., and Dodds, J. A. 1998. Naturally occurring variants of satellite tobacco mosaic virus. Phytopathology 88:514-519.

Four natural variants of satellite tobacco mosaic virus (STMV) were compared with each other and with the type strain. Differences were detected in double-stranded RNA, single-stranded RNA, and virion electrophoretic mobility patterns, while the size and antigenicity of the coat protein were similar for all. RNase protection assays detected differences in the genomes of each of the four new variants, which differed not only from each other, but also from that of type STMV.
Infectious RNA transcripts were made from complementary DNA clones of one variant (STMV 10) with a genome apparently smaller than that of type STMV. A 71-base deletion in the region that contains the $6.8-\mathrm{kDa}$ protein in type STMV was detected by sequence analysis of the STMV 10 clones, a result that is confirmed by the lack of a $6.8-\mathrm{kDa}$ in vitro translation product for STMV 10. Only minor sequence differences exist elsewhere in the genome compared with that of type STMV. Type STMV and STMV 10 each successfully cross-protected against the other when tobacco plants were inoculated 10 days apart.
Satellite tobacco mosaic virus (STMV) was discovered in 1986 in plants of Nicotiana glauca Graham (tree tobacco) that were also infected with tobacco mild green mosaic virus (TMGMV, also known as TMV-U5) (21,22). STMV is a 17-nm, spherical virus with $\mathrm{T}=1$ symmetry (6) and is dependent upon tobamoviruses for its replication. It produces its own 17.5-kDa coat protein from a plus-sense, single-stranded (ss) RNA genome of 1,059 nucleotides (14). An overlapping open reading frame (ORF) for a $6.8-\mathrm{kDa}$ protein of unknown function is present upstream of the coat protein gene in the genome of the type strain (14). The first isolate of STMV to be cloned and sequenced was designated the "type" strain (14), but it has become apparent through genome comparisons in which RNase protection assays (RPAs) were used that STMV isolates are genetically quite diverse $(8,9)$ and that a dominant "strain" may not exist in nature. However, few of these isolates were fully sequenced or characterized for other viral properties so that a broad comparison could be performed. The present study was initiated to better understand variability among isolates of STMV and to evaluate whether the type strain is representative of the virus.

Four field isolates of STMV among a collection of isolates from individual plants of $N$. glauca infected with TMGMV in Riverside County, CA, were identified by double-stranded (ds) RNA electrophoretic mobilities as different from type STMV (three had larger dsRNA, and one had smaller). They were originally isolated by R. Valverde, who provided them for use in this study. The isolates, designated STMV 6, 8, 10, and 19 (isolate numbers are not the same as those used by others, including Kurath et al. [8,9] and Valverde and Dodds [22]), were studied for general viral characters, and one was chosen for cloning and sequencing. Analysis of these isolates has provided additional information on the natural diversity of STMV, including evidence for the dispensability of the $6.8-\mathrm{kDa}$ protein.

\section{MATERIALS AND METHODS}

Virus isolates. The four variants (STMV 6, 8, 10, and 19) were activated by hydrating and grinding $100 \mathrm{mg}$ of dried tissue in $300 \mu \mathrm{l}$

Corresponding author: D. M. Mathews; E-mail address: dmathews@ucrac1.ucr.edu

Publication no. P-1998-0331-01R

(C) 1998 The American Phytopathological Society of $0.02 \mathrm{M}$ potassium phosphate buffer, $\mathrm{pH} 7.2$ (inoculation buffer). The extract was used to inoculate $N$. tabacum L. cv. Xanthi tobacco plants (50 $\mu \mathrm{l}$ per leaf, two leaves per plant for each isolate). Type STMV inoculum was prepared by using the extract obtained from grinding $1 \mathrm{~g}$ of frozen plant tissue in $1 \mathrm{ml}$ of inoculation buffer, and plants were similarly inoculated. Plants were incubated in growth chambers (one isolate per chamber) for 21 days at $25^{\circ} \mathrm{C}$ with $14 \mathrm{~h}$ of light per day. Tissue was then harvested and used for various assays.

dsRNA isolation. dsRNAs were purified from $7 \mathrm{~g}$ of tissue by using the standard procedure of Morris and Dodds (15), analyzed by electrophoresis through $6 \%$ polyacrylamide mini-slab gels, and stained with ethidium bromide for detection and photography.

Virus particle purification and virion analysis. Fifty grams of STMV-infected tissue was harvested, and virus was purified as previously described (23). Approximately $5 \mu \mathrm{g}$ of partially purified (pregradient) virus from each of the four variants as well as type STMV was separated by electrophoresis through a $1 \%$ agarose gel for $1 \mathrm{~h}$ at $8 \mathrm{~V} / \mathrm{cm}$. TAE (40 mM Tris-Cl, $20 \mathrm{mM}$ sodium acetate, and 1mM EDTA, pH 7.4) was used as the running buffer. The gel was stained with ethidium bromide and photographed.

Coat protein analysis. Gradient-purified virus was denatured by boiling it for $2 \mathrm{~min}$ in a protein dissociation buffer $(62 \mathrm{mM}$ Tris-Cl, $\mathrm{pH}$ 6.8, 2\% sodium dodecyl sulfate [SDS], 5\% 2-mercaptoethanol, and $10 \%$ glycerol) and then analyzed by electrophoresis through a $15 \%$ polyacrylamide- $0.1 \%$ SDS gel (SDSPAGE) (10). The gel was stained overnight with Coomassie blue $\mathrm{R}-250$, destained for $2 \mathrm{~h}$ with several changes of $50 \%$ methanol plus $10 \%$ acetic acid, destained with $5 \%$ methanol plus $7 \%$ acetic acid until the background was clear, and then photographed.

Ouchterlony double-diffusion assays (22) were used to check for antigenic differences. A preparation of type STMV was applied to alternating wells adjacent to each new variant, and the plates were observed daily for 4 days to check for spur formation.

Purification and analysis of ssRNA from virions. ssRNA was isolated from gradient-purified STMV by using a proteinase $\mathrm{K}$ procedure (13). Purified ssRNA (500 ng) was denatured and loaded onto a $1 \%$ agarose gel containing $1.1 \%$ formaldehyde and subjected to electrophoresis at $5 \mathrm{~V} / \mathrm{cm}$ for $2 \mathrm{~h}$ with $40 \mathrm{mM}$ MOPS (3-[N-morpholino]propanesulfonic acid), pH 7.0, $10 \mathrm{mM}$ sodium acetate, and $1 \mathrm{mM}$ EDTA as the running buffer (1). The gel was stained with ethidium bromide and photographed. 
One microgram of STMV ssRNA was used in in vitro translation reactions in either a wheat germ extract or a rabbit reticulocyte cell-free system (Promega Biotec, Madison, WI) with ${ }^{35} \mathrm{~S}$ labeled methionine. Translation products $(150,000 \mathrm{cpm}$ of each) were boiled in protein dissociation buffer, loaded onto SDS-PAGE gels, and analyzed as described above. The proteins were fixed by washing the gel for $3 \mathrm{~h}$ with several changes of $30 \%$ methanol plus $10 \%$ acetic acid. The gel was vacuum dried at $60^{\circ} \mathrm{C}$ and exposed to Kodak X-Omat AR 5 film for 24 to $72 \mathrm{~h}$.

RPAs. Approximately $100 \mathrm{ng}$ of single-stranded virion RNA or $500 \mathrm{ng}$ of dsRNA, which had been denatured by boiling it for $3 \mathrm{~min}$, was used as target RNA in the analyses. Complementary DNA constructs of the STMV genome in the transcription vector pBluescribe (pBS; Stratagene, San Diego, CA) were used to produce minus-sense (T3) RNA transcripts for use as probes in heterogeneity mapping $(9,24)$. Conditions of probe production, hybridization, and gel analysis were identical to those of Kurath et al. (9), except that the $6 \%$ PAGE gels were dried under vacuum at $80^{\circ} \mathrm{C}$ prior to autoradiography.

cDNA cloning and sequencing. Complementary DNA clones were produced from purified ssRNA of STMV 10 by using the primers and methods described previously (9). After screening and selection based on the size of the inserts, plasmid DNA from each of five clones was denatured and sequenced with the Sequenase enzyme system (United States Biochemical, Cleveland, $\mathrm{OH})$ to perform dideoxynucleotide termination reactions (20). Sequencing primers included both the forward and reverse M13 primers (Amersham, Arlington Heights, IL) and an STMV-specific oligonucleotide complementary to nucleotides 797 to 815 .

Infectivity of in vitro RNA transcripts. Plasmid DNA from each of the five STMV 10 clones was linearized and blunt-ended (9) and then used to direct the synthesis of genomic sense RNA transcripts by using T7 RNA polymerase (Promega Biotec) in $100-\mu \mathrm{l}$ reactions according to the manufacturer's instructions. After transcription, $700 \mu \mathrm{l}$ of RNA inoculation buffer $(0.05 \mathrm{M}$ glycine, $0.03 \mathrm{M} \mathrm{K}_{2} \mathrm{HPO}_{4}, \mathrm{pH} 9.2,1 \%$ sodium pyrophosphate, $1 \%$ macaloid [NL Chemicals, Hightstown, NJ], and $1 \%$ Celite) was added, and the mixture was used to inoculate tobacco plants $(N$. tabacum cv. Xanthi; $50 \mu \mathrm{l}$ per leaf, two leaves per plant), which had been inoculated with TMGMV ssRNA or virions $24 \mathrm{~h}$ previously. Plants were incubated for 14 days in a growth chamber at $25^{\circ} \mathrm{C}$ and then tested for the presence of STMV by Ouchterlony immunodiffusion assays and dsRNA analysis.

Cross-protection between type STMV and STMV 10. $N$. tabacum cv. Xanthi plants were inoculated with the helper virus TMGMV and then $24 \mathrm{~h}$ later with RNA transcripts of either type STMV or STMV 10 as the protecting strain. After 10 days, plants were inoculated with RNA transcripts of either STMV 10 or type STMV as the challenge strain and incubated for an additional 14 days. Controls consisted of plants inoculated with TMGMV alone, plants with single and double infections of type STMV and STMV 10 in the presence of TMGMV, and mock-inoculated plants. Plants were assayed by dsRNA analysis as described above.

\section{RESULTS}

Analysis of nucleic acids. Compared with that of type STMV, the dsRNAs of three of the variants (STMV 6, 8, and 19) had slower electrophoretic mobilities on $6 \%$ polyacrylamide gels and one dsRNA (STMV 10) had faster mobility, indicating a larger or smaller genome than that of type STMV, respectively (Fig. 1A). dsRNAs of all strains appeared to accumulate to similar levels in tobacco plants. When ssRNAs from virions were analyzed by agarose gel electrophoresis under denaturing conditions, those from STMV 6, 8, and 19 all migrated to the same position in the gel as the type STMV, while STMV 10 ssRNA migrated slightly faster than that of type STMV, indicating that it was smaller (Fig. 1B).
When ssRNA from each of the five STMV isolates was used to direct protein translation in an in vitro wheat germ extract system, STMV 6 produced both the coat protein and the $6.8-\mathrm{kDa}$ product, a result identical to that for the ssRNA of type STMV (Fig. 2, lanes 6 and T). The other three isolates produced the coat protein $(17 \mathrm{kDa}$ ) but not the 6.8-kDa protein product (Fig. 2, lanes 8, 10, and 19). Results were identical when ssRNAs were used in a rabbit reticulocyte cell-free translation system (results not shown).

Virion and coat protein analysis. The electrophoretic mobilities of intact virions from type STMV and STMV 8 and 19 were similar (Fig. 3, lanes T, 8, and 19). The mobilities of STMV 6 and 10 virions were similar to each other but different from the other three, migrating further from the origin (Fig. 3, lanes 6 and 10).

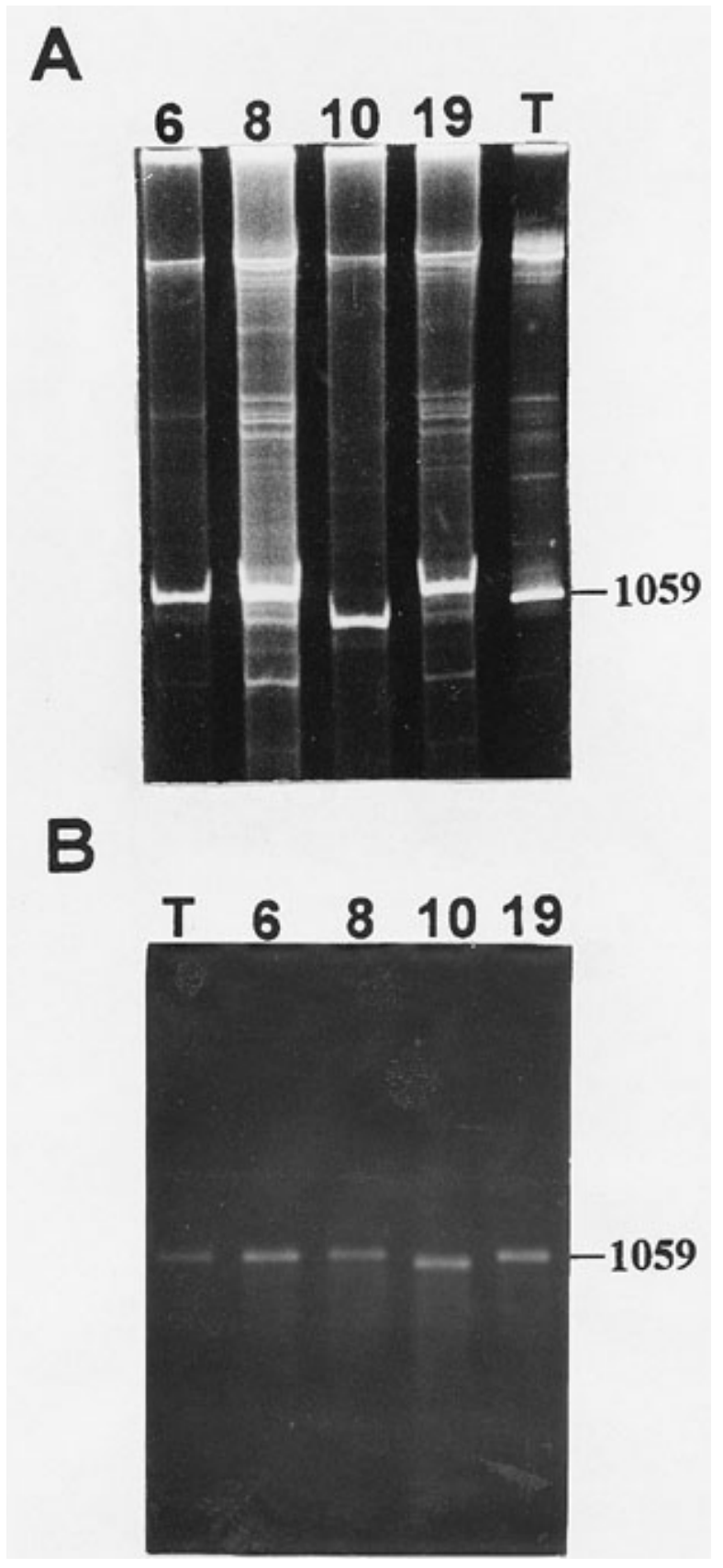

Fig. 1. A, Double-stranded (ds) RNAs extracted from tobacco tissue infected with variants of satellite tobacco mosaic virus (STMV) and tobacco mild green mosaic virus and analyzed by $6 \%$ polyacrylamide gel electrophoresis and B, single-stranded RNAs extracted from gradient purified virions of STMV variants analyzed by $1 \%$ agarose- $1.1 \%$ formaldehyde denaturing gel electrophoresis. STMV isolate numbers are shown above each lane, and nucleotide lengths are shown at the side. The amount of dsRNA loaded in each lane was equivalent to $0.7 \mathrm{~g}$ of tissue (1/10 of total extract). 
In order to compare the sizes of the isolates' coat proteins, denatured coat proteins were analyzed by SDS-PAGE and stained. The coat proteins from all five isolates migrated to the same position in the gel (results not shown). Ouchterlony double-diffusion tests showed no antigenic differences among the coat proteins of the four new variants and type STMV. All isolates produced one clear precipitin line, and no spur formation occurred between any of the isolates and type STMV (results not shown).

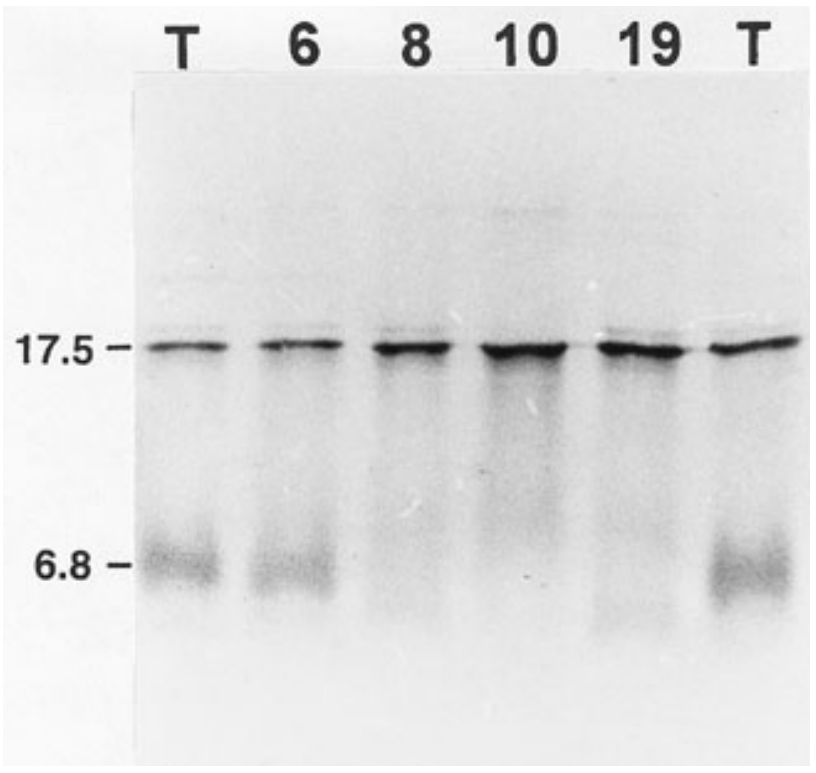

Fig. 2. Autoradiograph of in vitro translation products analyzed by electrophoresis through a $15 \%$ polyacrylamide- $0.1 \%$ sodium dodecyl sulfate gel. Identity of single-stranded RNAs from satellite tobacco mosaic virus isolates used to direct protein synthesis are shown above each lane, and protein molecular weights $\left(\times 10^{3} M_{\mathrm{r}}\right)$ of the coat protein (17.5) and 6.8-kDa protein are indicated on the left.

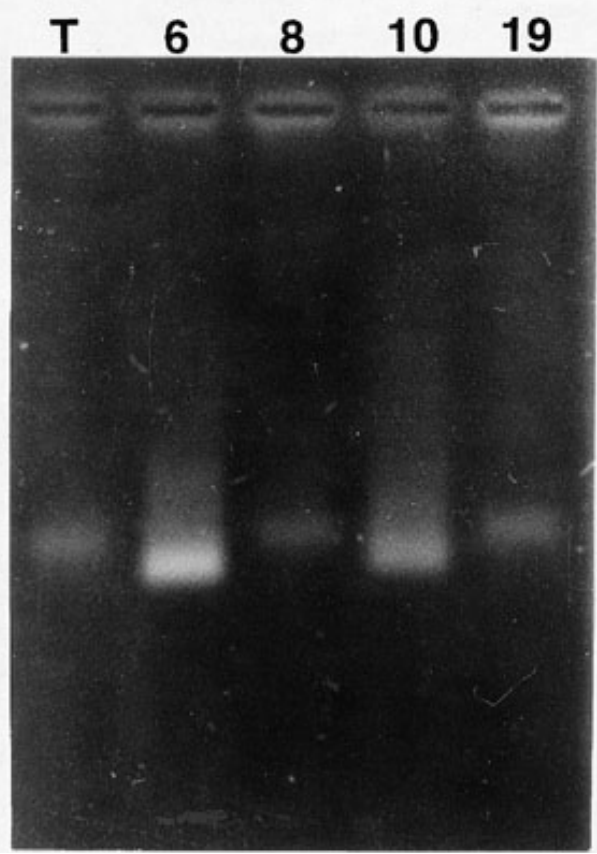

Fig. 3. Intact virions of satellite tobacco mosaic virus (STMV) variants analyzed by $1 \%$ agarose gel electrophoresis and stained with ethidium bromide. STMV isolate numbers are shown above each lane.
RPAs. Two overlapping minus-sense RNA probes were used in the assays, one covering the $5^{\prime} 791$ terminal nucleotides (probe B) and one covering the $3^{\prime} 457$ terminal nucleotides (probe A) of the 1,059-nucleotide genome. The RNase digestion patterns of the heteroduplexes formed between the genomic RNAs of the variants and the two probes were compared by using both virion ssRNA and denatured dsRNA as the target molecules in the assays. There were no differences in the banding patterns obtained with ssRNA

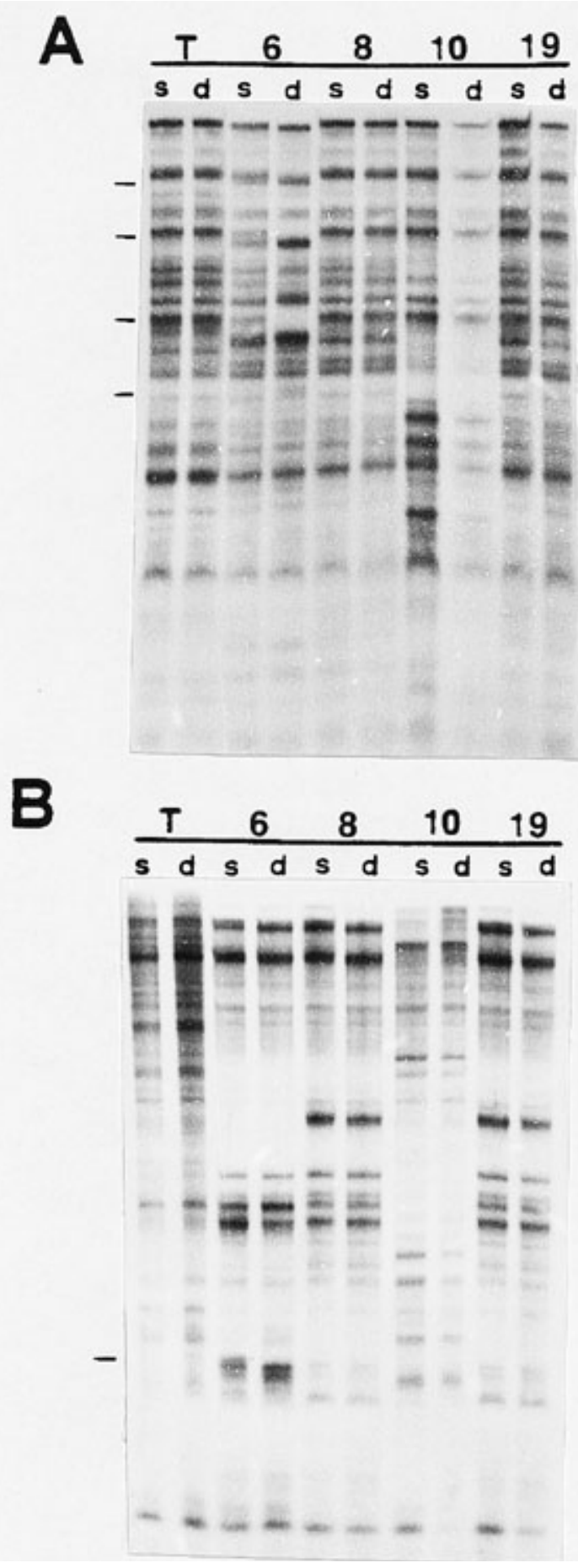

Fig. 4. Results of RNase protection assays using two overlapping minus-sense RNA transcript probes covering the 457 3' nucleotides (A) and the $7915^{\prime}$ terminal nucleotides (B) of type satellite tobacco mosaic virus (STMV). Target RNAs were single-stranded (ss) RNAs (s) or denatured double-stranded (ds) RNAs (d) from type STMV (T) or from STMV 6, 8, 10, or 19 (labeled appropriately). Positions of bands that differ between the ssRNA and dsRNA results for STMV 6 are marked along the left. 
or dsRNA for type STMV or STMV 8, 10, or 19 (Fig. 4). However, the denatured dsRNA banding pattern of STMV 6 was slightly different from the pattern for ssRNA (Fig. 4, lanes 6s and d).

The RNase digestion patterns indicate that each of the new variants differed significantly from type STMV at the $5^{\prime}$ end (probe B) but were quite similar at the $3^{\prime}$ end (probe A) (Fig. 4).
STMV 8 and 19 appeared to be identical in their banding patterns for both probes $\mathrm{A}$ and $\mathrm{B}$, regardless of whether ssRNA or dsRNA was used as the target molecule (Fig. 4A and B, lanes 8 and 19). STMV 10 varied the most from the other strains on the basis of results from using probe B (Fig. 4 and B, lanes 10s and d).

Cloning and sequencing of STMV strains. On the basis of the results of the other assays, STMV 10 was chosen for cloning and

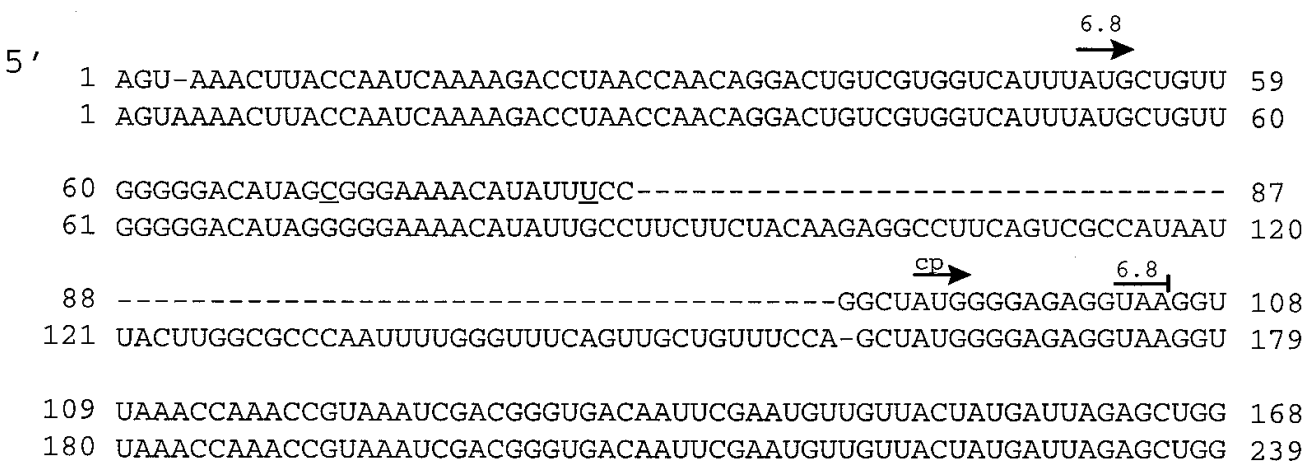

169 AAGCUAUCCUAAGGUCAAUCCGACUCCAACGUGGGUCAGAGCCAUACCUUUCGAAGUGUC 228 240 AAgCUAUCCUAAGGUCAAUCCGACUCCAACGUGGGUCAGAGCCAUACCUUUCGAAGUGUC 299

229 AGUUCAAUCUGGUAUUGCUUUUAAAGUACCGGUCGGGUCACUAUUUUCGGCAAAUUUCCG 288 300 AgUUCAAUCUGGUAUUGCUUUUAAAGUACCGGUCGGGUCACUAUUUUCGGCAAAUUUCCG 359

289 GACAGAUUCCUUUACAAGCGUCACAGUGAUGAGUGUCCGUGCUUGGACCCAGUUAACACC 348 360 GACAGAUUCCUUUACAAGCGUCACAGUGAUGAGUGUCCGUGCUUGGACCCAGUUAACACC 419

349 GCCAGUAAAUGAGUACAGUUUUGUGAGGCUGAAGCCAUUGUUCAAGACUUGUGACUCUAC 408 420 GCCAGUAAAUGAGUACAGUUUUGUGAGGCUGAAGCCAUUGUUCAAGACUUGUGACUCUAC 479

409 UGAGGAGUUCGAAGGGCGUGCAUCAAACAUCAACACACGAGCUUCUGUAGGGUACAGGAU 468 480 UGAGGAGUUCGAAGGGCGUGCAUCAAACAUCAACACACGAGCUUCUGUAGGGUACAGGAU 539

469 UCCAACUAAUUUGCGUCAGAAUACUGUGGCAGCCGACAAUGUAUGCGAAGUAAGAAGUAA 528 540 UCCAACUAAUUUGCGUCAGAAUACUGUGGCAGCCGACAAUGUAUGCGAAGUAAGAAGUAA 599 529 CUGUCGACAAGUCGCCUUGGUUAUUUCGUGUUGUUUUAACUGAACCUGCACAUAAGCCUU 588 600 CUGUCGACAAGUCGCCUUGGUUAUUUCGUGUUGUUUUAACUGAACCUGCACAUAAGCCUU 659

589 UUAAGAUCGAAGGUUAAACGAUCUGGCUCCUCGCUUGAGCUUGAGGCGGCGUAUCUCUUAUG 648 660 UUGGAUCGAAGGUUAAACGAUCCGCUCCUCGCUUGAGCUUGAGGCGGCGUAUCUCUUAUG 719

649 UCAACAGAGACACUUUGGUCUAUGGUUGUAUAACAAUAGAUAGACUCCCGUUUGCAAGAU 708 720 UCAACAGAGACACUUUGGUCUAUGGUUGUAUAACAAUAGAUAGACUCCCGUUUGCAAGAU 779

709 UAGgGUUAACAGAUCUUGCCGUUAGÇCUGGUUAGCGCGUAACCGGCCUUGAUUUAUGGAA 768 780 UAGGGUUAACAGAUCUUGCCGUUAGUCUGGUUAGCGCGUAACCGGCCUUGAUUUAUGGAA 839

769 UAGAUCCAUUGUCCAAUGGGCUUUGCCAAUGGAACGCCGACGUGGCUGUAUAAUACGUCG 828 840 UAGAUCCAUUGUCCAAUGG-CUUUGCCAAUGGAACGCCGACGUGGCUGUAUAAUACGUCG 898

829 UUGACAAGUACGAAAUCUUGUUAGUGUUUUUCCCUCCACUUAAAUCGAAGGGUUUUGUUU 888 899 UUGACAAGUACGAAAUCUUGUUAGUGUUUUUCCCUCCACUUAAAUCGAAGGGUUUUGUUU 958

889 UGGUCUUCCCGAACGCAUACGUUAGUGUGACUACCGUUGUUCGAAACAAGUAAAACAGGA 948 959 UGGUCUUCCCGAACGCAUACGUUAGUGUGACUACCGUUGUUCGAAACAAGUAAAACAGGA 1018

949 AGGGGGUUCGAAUCCCUCCCUAACCGCGGGUAAGCGGCCCA 989 1019 AGGGGGUUCGAAUCCCUCCCUAACCGCGGGUAAGCGGCCCA 1059

Fig. 5. Nucleotide sequence (genomic sense) of satellite tobacco mosaic virus (STMV) 10 RNA (upper lines) compared with type STMV RNA (lower lines). Insertions and deletions are noted by hyphens, and single base changes are underlined. Start $(\rightarrow)$ and stop $(-t)$ codons are shown for the coat protein $(\mathrm{cp})$ and the 6.8-kDa protein. 
sequencing, since it varied the most from type STMV for all characters tested (except coat protein size) and its smaller genome size allowed for easy comparison in gel electrophoresis.

After cloning and screening, five full-length clones of STMV 10 were recovered and designated pDM10-43, -48, -65, -66, and -75. Each clone was sequenced, and a consensus was derived and compared with that of the type strain (Fig. 5). One of the five clones (pDM10-65) had an aberrant sequence in the $3^{\prime}$ half of its genome that did not match the consensus. Compared with the published sequence of type STMV, STMV 10 had a major deletion between bases 89 and 159 (inclusive) that removed most of the ORF for the $6.8-\mathrm{kDa}$ protein. There were eight additional single-base differences, additions, or deletions in the genome of STMV 10 that distinguish it from type STMV (Fig. 5). The coat protein gene was fully conserved. The total length of the STMV 10 genome is 989 nucleotides compared with 1,059 nucleotides of type STMV.

Infectivity of STMV 10 RNA transcripts. STMV 10 transcripts from cDNA clones pDM10-43, $-48,-66$, and -75 were all infectious when inoculated onto tobacco plants in the presence of TMGMV, as determined by Ouchterlony double-diffusion assays. Transcripts from pDM10-65 were not infectious. dsRNA isolated from plants infected with each of the four infectious transcripts migrated as expected in $6 \%$ polyacrylamide gels, with an electrophoretic mobility faster than that of type STMV (data not shown).

Cross-protection and coinoculation. RNA transcripts from both type STMV and STMV 10 failed to superinfect tobacco plants previously inoculated with the other strain and TMGMV (Fig. 6). When coinoculated onto a tobacco plant with TMGMV, each strain was able to accumulate to similar levels on the basis of dsRNA analysis (Fig. 6, lane 6). dsRNAs extracted from all control plants migrated as expected.

\section{DISCUSSION}

dsRNA analysis was the approach that allowed initial identification of the uniqueness of the strains compared in this study.

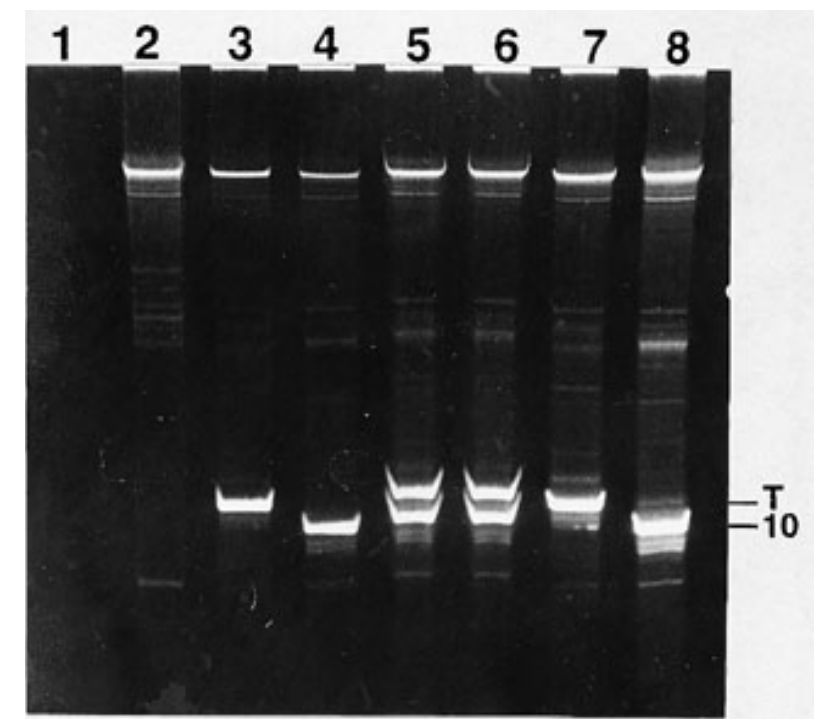

Fig. 6. Cross-protection between strains: $6 \%$ polyacrylamide gel electrophoresis of double-stranded (ds) RNAs that accumulated after inoculation with buffer only (lane 1); tobacco mild green mosaic virus (TMGMV) only (lane 2); TMGMV and type satellite tobacco mosaic virus (STMV) (lane 3); TMGMV and STMV 10 (lane 4); a physical mixture of samples from lanes 3 and 4 (lane 5); TMGMV, type STMV, and STMV 10 (coinoculated) (lane 6); TMGMV and type STMV followed 10 days later by STMV 10 (lane 7); and TMGMV and STMV 10 followed 10 days later by type STMV (lane 8). The amount of dsRNA loaded in each lane was equivalent to $0.7 \mathrm{~g}$ of tissue $(1 / 10$ of total extract). The positions of the replicative dsRNAs are marked for type STMV (T) and STMV 10 (10).
Although dsRNA from each of the new isolates varied from type STMV in electrophoretic mobility, only the ssRNA from STMV 10 had a significantly different mobility under denaturing conditions. Therefore, the differences in the dsRNAs from the variants with slower mobilities are probably not the result of a larger genome size, but of conformational differences of the doublestranded molecules.

No antigenic differences were detected between any of the new variants and type STMV. STMV 10, for which the complete RNA sequence was derived, had no nucleotide differences within the coat protein gene compared with the published sequence of the type strain. RPAs have been used to show that many field isolates of STMV exhibit great diversity within the coat protein gene (8), but this is not the case with STMV 10. Since there is apparently little or no difference in the size or primary structure of the coat protein, the differences in virion mobility may result from overall charge or size differences in virions contributed by the internal RNA. The RNA of type STMV is known to have double-helical regions that are intimately associated with the capsid protein subunits within the virions of type STMV $(11,12)$. It is thought that these RNA-protein interactions may be important for particle structure and may help explain the different mobility patterns.

Although RPAs can be used to map a genome $(9,24)$, the purpose for using them with these isolates of STMV was simply to show overall differences among them. Few differences were observed among the five variants when the probe covering the $3^{\prime}$ 457 terminal nucleotides (probe A) was used. Some minor variations were detected for STMV 6 and 10 in this region. When the probe covering the $5^{\prime} 791$ terminal nucleotides (probe B) was used, major differences were found among variants, except between STMV 8 and 19, which were identical to each other but had digestion patterns different from those of the other three isolates. These results agree with previously published data that show most of the genetic diversity of STMV occurs in the $5^{\prime}$ half of its genome (8).

There was little difference between ssRNA or denatured dsRNA as the experimental target RNA for RPAs. This has also been noted when the satellite RNA (CARNA 5) of cucumber mosaic virus was used (G. Kurath, personal communication). The use of nucleic acids purified at different times from individual viral infections could be responsible for the small differences seen between ssRNAs and dsRNAs of STMV 6 in RPAs. The use of dsRNA for RPAs of plant viruses has not been exploited. This study indicates that there is value in using this readily obtained product of viral infection for studies of genome diversity.

The 71-base deletion in STMV 10 results in the termination of the ORF for the 6.8-kDa protein after 51 nucleotides (17 amino acids, an approximately $1.5-\mathrm{kDa}$ protein). This agrees with the fact that no $6.8-\mathrm{kDa}$ product is produced in in vitro translation. This shorter genome length also does not appear to interfere with packaging of the RNA into virions, although with almost $10 \%$ of the genome missing compared with that of type STMV, one can assume that some differences must exist. Initial studies in which X-ray crystallography was used did not detect any differences between STMV 10 virions and type STMV (A. McPherson, personal communication). Experimental manipulation of the 6.8$\mathrm{kDa}$ ORF has shown that when it is compromised in cDNA clones of type STMV, there is no effect on replication or accumulation of the virus in tobacco plants (17). The apparent dispensability of this gene in artificially created mutants has now been supported by analysis of natural variants, and we conclude that the $6.8-\mathrm{kDa}$ product is a nonessential protein for replication or field transmission of these isolates. The production of infectious clones of STMV 10 ruled out the possibility that low levels of the $6.8-\mathrm{kDa}$ protein were being produced by an undetected infection of an isolate that did contain the ORF for this protein. Similarly, small ORFs that are translated in vitro have also been reported in most isolates of CARNA $5(2,16)$, but associated proteins have not been 
found in vivo, and the presence of the ORFs does not affect the biological function of the satellite (3-5). The presence of putative small ORFs without associated in vitro translational function have been reported for satellite RNAs of the tombusvirus and nepovirus groups $(18,19)$.

Infections resulting from RNA transcripts of both type STMV and STMV 10 were able to fully cross-protect against each other in tobacco plants when a 10-day waiting period was used between protection and challenge inoculations. Shorter protection periods were not tested in this study, but when two different isolates of STMV that varied by only five single nucleotides were used, it was shown that when the interval was less than $24 \mathrm{~h}$, superinfection of the challenging strain could occur (7). Both type STMV and STMV 10 were able to accumulate when coinoculated.

Previous comparisons of diversity between natural STMV isolates have relied heavily on RPAs. This study was conducted in order to make a broader comparison by using a variety of techniques. STMV 6, like type STMV, was able to translate the 6.8$\mathrm{kDa}$ protein of STMV in vitro, implying that such strains are to be found in nature. However, variants differing from the "type" strain, including some that lack or cannot express the $6.8-\mathrm{kDa}$ protein coding sequence, may also be common. This study raises questions about the importance, frequency, and function of the $6.8-\mathrm{kDa}$ protein in natural infections, and this is now being investigated.

\section{LITERATURE CITED}

1. Ausubel, F. M., Brent, R., Kingston, R. E., Moore, D. D., Seidman, J. G., Smith, J. A., and Struhl, K. 1987. Preparation and analysis of RNA. Pages 4.9.1-4.9.4 in: Current Protocols in Molecular Biology. John Wiley \& Sons, New York.

2. Avila-Rincon, M. J., Collmer, C. W., and Kaper, J. M. 1986. In vitro translation of cucomoviral satellites. II. CARNA 5 from cucumber mosaic virus strain S and SP6 transcripts of cloned (S)CARNA 5 cDNA produce electrophoretically comigrating protein products. Virology 152 : 455-458.

3. Collmer, C. W., and Kaper, J. M. 1988. Site-directed mutagenesis of potential protein-coding regions in expressible cloned cDNAs of cucumber mosaic viral satellites. Virology 163:293-298.

4. Garcia-Arenal, F., Zaitlin, M., and Palukaitis, P. 1987. Nucleotide sequence analysis of six satellite RNAs of cucumber mosaic virus: Primary sequence and secondary structure alterations do not correlate with differences in pathogenicity. Virology 158:339-347.

5. Jaegle, M., Devic, M., Longstaff, M., and Baulcombe, D. 1990. Cucumber mosaic virus satellite RNA (Y strain): Analysis of sequences which affect yellow mosaic symptoms on tobacco. J. Gen. Virol. 71:1905-1912.

6. Koszelak, S., Dodds, J. A., and McPherson, A. 1989. Preliminary analysis of crystals of satellite tobacco mosaic virus. J. Mol. Biol. 209:323-325.

7. Kurath, G., and Dodds, J. A. 1994. Satellite tobacco mosaic virus se- quence variants with only five nucleotide differences can interfere with each other in a cross protection-like phenomenon in plants. Virology 202: 1065-1069.

8. Kurath, G., Heick, J. A., and Dodds, J. A. 1993. RNase protection analyses show high genetic diversity among field isolates of satellite tobacco mosaic virus. Virology 194:414-418.

9. Kurath, G., Rey, M. E. C., and Dodds, J. A. 1992. Analysis of genetic heterogeneity within the type strain of satellite tobacco mosaic virus reveals several variants and a strong bias for $\mathrm{G}$ to A substitution mutations. Virology 189:233-244.

10. Laemmli, U. K. 1970. Cleavage of structural proteins during the assembly of the head of the bacteriophage T4. Nature (London) 227:680685.

11. Larson, S. B., Koszelak, S., Day, J., Greenwood, A., Dodds, J. A., and McPherson, A. 1993. Double-helical RNA in satellite tobacco mosaic virus. Nature (London) 361:179-182.

12. Larson, S. B., Koszelak, S., Day, J., Greenwood, A., Dodds, J. A., and McPherson, A. 1993. Three-dimensional structure of satellite tobacco mosaic virus at $2.9 \AA$ A resolution. J. Mol. Biol. 231:375-391.

13. Maniatis, T., Fritsch, E. F., and Sambrook, J. 1982. Molecular Cloning: A Laboratory Manual. Cold Spring Harbor Laboratory, Cold Spring Harbor, NY

14. Mirkov, T. E., Mathews, D. M., Du Plessis, D. H., and Dodds, J. A. 1989. Nucleotide sequence and translation of satellite tobacco mosaic virus RNA. Virology 170:139-146.

15. Morris, T. J., and Dodds, J. A. 1979. Isolation and analysis of doublestranded RNA from virus-infected plant and fungal tissue. Phytopathology 69:854-858.

16. Owens, R. A., and Kaper, J. M. 1977. Cucumber mosaic virus-associated satellite RNA 5. II. In vitro translation in a wheat germ protein-synthesis system. Virology 80:196-203.

17. Routh, G., Dodds, J. A., Fitzmaurice, L., and Mirkov, T. E. 1995. Characterization of deletion and frameshift mutants of satellite tobacco mosaic virus. Virology 212:121-127.

18. Rubino, L., Burgyan, J., Grieco, F., and Russo, M. 1990. Sequence analysis of cymbidium ringspot virus satellite and defective interfering RNAs. J. Gen. Virol. 71:1655-1660.

19. Rubino, L., Tousignant, M. E., Steger, G., and Kaper, J. M. 1990. Nucleotide sequence and structural analysis of two satellite RNAs associated with chicory yellow mottle virus. J. Gen. Virol. 71:18971903.

20. Sanger, F., Nicklen, S., and Coulson, A. R. 1977. DNA sequencing with chain-terminating inhibitors. Proc. Natl. Acad. Sci. USA 74:5463-5467.

21. Valverde, R. A., and Dodds, J. A. 1986. Evidence for a satellite RNA associated naturally with the U5 strain and experimentally with the U1 strain of tobacco mosaic virus. J. Gen. Virol. 67:1875-1884.

22. Valverde, R. A., and Dodds, J. A. 1987. Some properties of isometric virus particles which contain the satellite RNA of tobacco mosaic virus. J. Gen. Virol. 68:965-972.

23. Valverde, R. A., Heick, J. A., and Dodds, J. A. 1991. Interactions between satellite tobacco mosaic virus, helper tobamoviruses, and their hosts. Phytopathology 81:99-104.

24. Winter, E., Yamamoto, F., Almoguera, C., and Perucho, M. 1985. A method to detect and characterize point mutations in transcribed genes: Amplification and over expression of the mutant c-Ki-ras allele in human tumor cells. Proc. Natl. Acad. Sci. USA 82:7575-7579. 